\title{
Exploring the infrared Landau gauge propagators using large asymmetric lattices
}

\author{
P. J. Silva* and O. Oliveira \\ Centro de Física Computacional, Departamento de Física, Universidade de Coimbra, \\ P-3004-516 Coimbra, Portugal \\ E-mail: psilva@teor.fis.uc.pt, orlando@teor.fis.uc.pt
}

\begin{abstract}
We report on the infrared limit of the quenched lattice Landau gauge gluon and ghost propagators computed from large asymmetric lattices. In particular, the compatibility of the pure power law infrared solutions of the Dyson-Schwinger equations with the lattice data is investigated and the exponent $\kappa$ is measured. The gluon lattice data favour $\kappa \sim 0.52$, which would imply a vanishing zero momentum gluon propagator. For the subset of lattices where the ghost propagator was computed, the data are not compatible with a pure power law. Our data also show a decreasing running coupling in the infrared region. Furthermore, positivity violation for the gluon propagator is also verified.
\end{abstract}

XXIVth International Symposium on Lattice Field Theory

July 23-28, 2006

Tucson, Arizona, USA

\footnotetext{
${ }^{*}$ Speaker.
} 


\section{Introduction and motivation}

The study of the infrared limit of Quantum Chromodynamics (QCD) requires the use of nonperturbative methods. Two first principles non-perturbative approaches are Dyson-Schwinger equations (DSE) and the lattice formulation of QCD. Because both methods have good and bad features, a comparison between their results is a good test of our understanding of the low energy limit of QCD. During the last years, there has been an effort to compute the infrared gluon and ghost propagators in Landau gauge,

$$
\begin{aligned}
D_{\mu v}^{a b}(q) & =\delta^{a b}\left(\delta_{\mu v}-\frac{q_{\mu} q_{v}}{q^{2}}\right) D\left(q^{2}\right), \\
G^{a b}(q) & =-\delta^{a b} G\left(q^{2}\right)
\end{aligned}
$$

and a running coupling constant ${ }^{1}$ defined from these propagators

$$
\alpha_{S}\left(q^{2}\right)=\alpha_{S}\left(\mu^{2}\right) Z_{\text {ghost }}^{2}\left(q^{2}\right) Z_{\text {gluon }}\left(q^{2}\right) ;
$$

$Z_{\text {ghost }}\left(q^{2}\right)=q^{2} G\left(q^{2}\right)$ and $Z_{\text {gluon }}\left(q^{2}\right)=q^{2} D\left(q^{2}\right)$ are the ghost and gluon dressing functions.

In [1], assuming ghost dominance, it was computed a solution of the DSE that predicts pure power laws for the propagators, namely

$$
Z_{\text {gluon }}\left(q^{2}\right) \sim\left(q^{2}\right)^{2 \kappa}, Z_{\text {ghost }}\left(q^{2}\right) \sim\left(q^{2}\right)^{-\kappa},
$$

with $\kappa=0.595$. This implies a vanishing (infinite) gluon (ghost) propagator for zero momentum. Other studies of the infrared limit also predict $\kappa>0.5[2,3,4]$.

As an infrared analytical solution of the DSE, the pure power laws are valid only for very low momenta. Indeed, comparing the DSE solution for the gluon propagator [5] with the corresponding pure power law, see figure 1, it comes out that the power law is valid only for momenta below 200 $\mathrm{MeV}$.

For lattice QCD, it is a challenge to perform a simulation with a minimum number of points in the region of interest. The symmetric lattices available at the moment have a limited number of points in the infrared region (see, for example, $[13,22,25]$ ).

In the series of papers $[6,7,8,9,10,11]$ we have been using large asymmetric lattices $L_{s}^{3} \times L_{t}$, with $L_{t} \gg L_{s}$, to investigate the infrared limit of the gluon and ghost propagators. In this article we report on the status of our investigations concerning the use of asymmetric lattices to study the infrared properties of QCD.

\section{Gluon propagator}

In [9], we have computed the gluon propagator for $\mathrm{SU}(3)$ four-dimensional asymmetric lattices $L_{s}^{3} \times 256$, with $L_{s}=8,10, \ldots, 18$.

\footnotetext{
${ }^{1}$ See [23] for a recent review on the running coupling constant.
} 


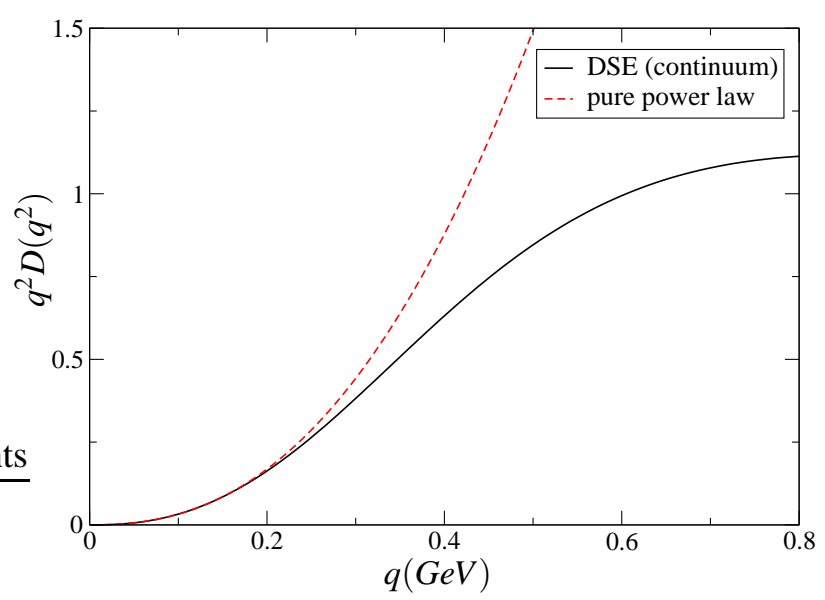

Figure 1: The gluon DSE solution [5] compared with the corresponding pure power law.


Figure 2: On the left, the gluon propagator for $16^{3} \times 128$ and $16^{3} \times 256$ lattices, considering only pure temporal momenta. Note the logarithmic scale in the vertical axis. On the right, the gluon propagator for all lattices $L_{s}^{3} \times 256$. For comparisation, we also show the $16^{3} \times 48$ and $32^{3} \times 64$ propagators computed in [20].

As reported in $[8,9,10,11,12,13]$, there are clear finite volume effects. However, the various simulations performed up to now show that the approach to the infinite volume limit $L_{s} \rightarrow+\infty$ is smooth.

In what concerns the finite volume effects, our simulations suggest that the finite volume effects are essentially due to a relatively small spatial extension. Indeed, the gluon propagator was computed for $16^{3} \times 128$ and $16^{3} \times 256$, and the data are undistinguishable - see figure 2 (left). This result gives us confidence that the temporal size of our lattices is sufficiently large.

However, it was observed that the propagator depends on the spatial size of the lattice - see figure 2 (right). The gluon propagator decreases with the volume for the smallest momenta and increases with the volume for higher momenta.

\subsection{Infrared exponent}

In order to compute the infrared exponent $\kappa$ from the lattice data, we considered fits of the 
smallest temporal momenta of the gluon dressing function $Z_{\text {gluon }}\left(q^{2}\right)=q^{2} D\left(q^{2}\right)$ to a pure power law with and without polinomial corrections ${ }^{2}$. In general, the $\kappa$ values increase with the volume of the lattice. So, our $\kappa$ can be read as lower bounds in the infinite volume figure $\kappa_{\infty}$.

The gluon propagator can be extrapolated to $L_{s} \rightarrow+\infty$, as a function of the inverse of the volume, fitting each timelike momentum propagator separately, and assuming a sufficient number of points in the temporal direction. Several types of polinomial extrapolations were tried, using different sets of lattices, and we conclude that the data are better described by quadratic extrapolations of the data from the 4 th and 5 th largest lattices.

The values of $\kappa$ extracted from these extrapolated propagators are $\kappa=0.5215(29)$, with a $\chi^{2} /$ d.o.f. $=0.02$, using the largest 5 lattices in the extrapolation, and $\kappa=0.4979(66), \chi^{2} /$ d.o.f. $=$ 0.27 using the largest 4 lattices. Fitting the extrapolated data to the polinomial corrections to the pure power law, one gets higher values for $\kappa$. The first value $\kappa=0.5215(29)$ is on the top of the value obtained from extrapolating directly $\kappa$ as a function of the volume [9]. Note also that in [7, 11], we fitted the gluon temporal data for larger ranges of momenta, using other model functions, giving always values for $\kappa$ above 0.5 , supporting again an infrared vanishing gluon propagator. In conclusion, one can claim a $\kappa \in[0.49,0.53]$, with the lattice data favouring the right hand side of the interval.

\subsection{Positivity violation}

In QCD, the violation of reflection positivity for the Landau gauge gluon propagator means that the gluon cannot appear as a free asymptotic S-matrix state. This may be viewed as an indication of gluon confinement.

On the lattice, one can study positivity violation for the gluon propagator from the real space propagator,

$$
C(t) \sim \int_{-\infty}^{\infty} d p D(p, \vec{p}=0) \exp (-i p t) .
$$

Finding $C(t)<0$ for some $t$ is a sign of positivity violation.

Positivity violation for the gluon propagator has been observed for the Dyson-Schwinger gluon propagator [14], as well as for the propagator computed from symmetric lattices [24, 13]. Here we show the real space propagator computed from our asymmetric lattices, including in figure $3, C(t)$ for the two infinite volume extrapolations of the gluon propagator computed in [9].

We thereby confirm that positivity violation occurs for the gluon propagator. Our data show that the time for positivity violation to happen, decreases when the spatial lattice volume is increased. Furthermore, the infinite volume limit suggests that positivity violation shows up at $t \sim 1.5 \mathrm{fm}$. Previous studies show similar values $[13,14]$. Moreover, similarly to what was observed in [24], for large time separations our data show an oscilatory behaviour.

\section{Ghost propagator}

So far, we have computed the ghost propagator for our smallest lattices [10] $\left(10^{3} \times 256,12^{3} \times\right.$ $256,16^{3} \times 128$ ), using both a point source method [18] (we averaged over 7 different point sources

\footnotetext{
${ }^{2}$ The results can be seen in table II of [9].
} 

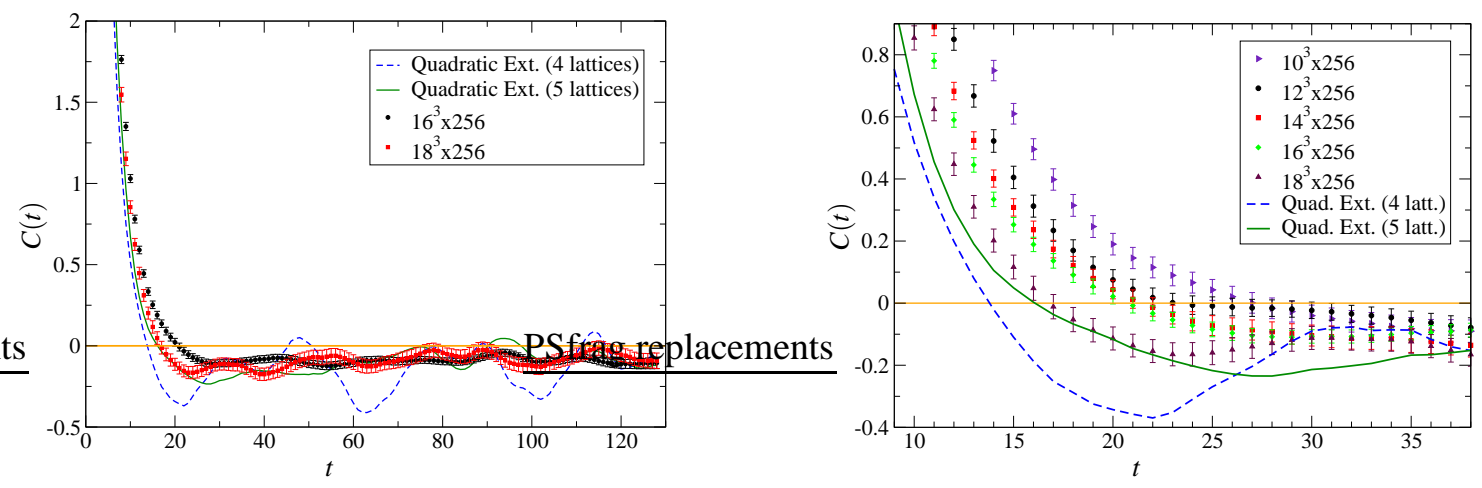

Figure 3: On the left, the real space gluon propagator $C(t)$ for our largest lattices and for the two extrapolations considered in [9]. On the right, a zoom of the region of interest showing all lattices.

to get a better statistics), and a plane-wave source [15]. In both cases we used the pre-conditioned conjugate gradient algorithm, as described in [16]. The plane-wave source method provides better statistical accuracy, but we can only obtain one momentum component at a time. The point source method allows to get all the momenta in one go, but with larger statistical errors.

In figure 4 (left) it is shown the ghost dressing function $Z_{\text {ghost }}\left(q^{2}\right)=q^{2} G\left(q^{2}\right)$ for the $16^{3} \times 128$ lattice, gauge fixed using CEASD method [17], computed with both methods. As in the gluon case, we can see differences, in the infrared, between pure temporal and pure spatial data. These differences vanish for sufficiently high momenta.


Figure 4: On the left, the bare ghost dressing function for the $16^{3} \times 128$ lattice, gauge fixed with CEASD method. "p2p" ("pws") stands for the ghost components computed using a point (plane wave) source. On the right, the bare ghost dressing function computed from a plane wave source for all lattices.

On the right hand side of figure 4, one can see the ghost dressing function only for the planewave data, for the available lattices. As in the gluon case [11], we are able to evaluate the effect of Gribov copies on the lattice $16^{3} \times 128$ by considering different gauge fixing methods. The data show clear effects of Gribov copies over a large range of momenta, as expected from other studies $[15,16]$. Also, we see finite volume effects if one compares propagators from lattices with different spatial sizes.

In what concerns the infrared region, we were unable to fit a pure power law, even considering 

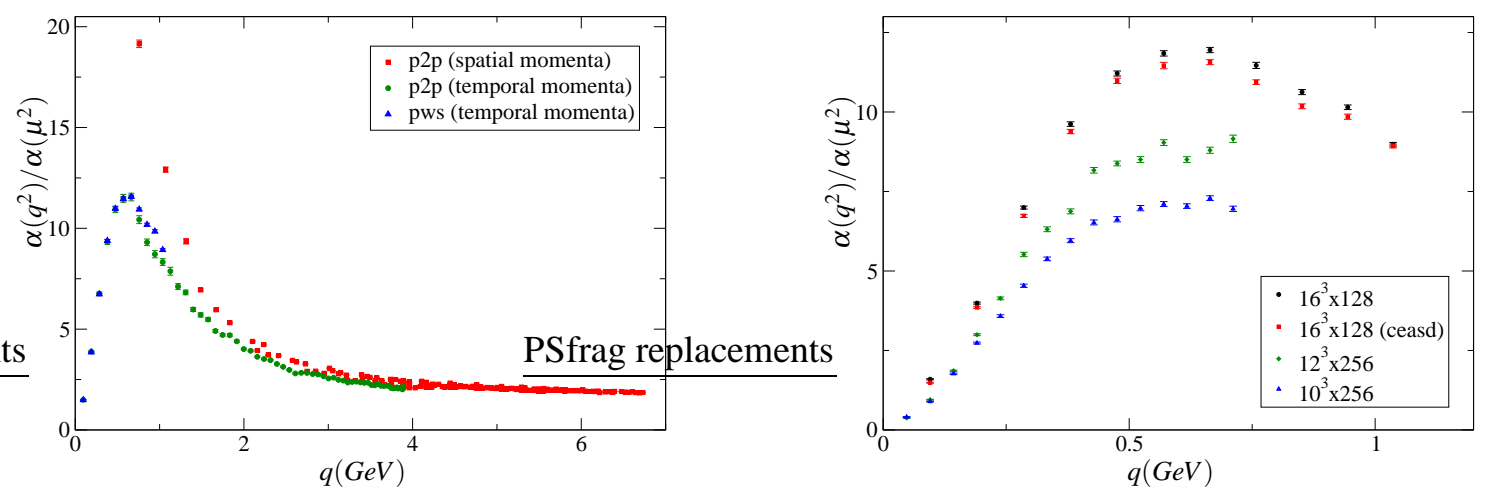

Figure 5: On the left, the running coupling constant for the $16^{3} \times 128$ lattice, gauge fixed with CEASD method. On the right, the running coupling constant for all lattices. The data were computed using a plane wave method.

polinomial corrections [10].

\section{Running coupling constant}

From the gluon and ghost dressing functions, one can define a running coupling constant see eq. 1.3.

The DSE infrared analysis predicts a running coupling constant at zero momentum different from zero, $\alpha_{S}(0)=2.972$ [1]. On the other hand, the DSE solution on a torus [19], and results from lattice simulations $[16,21,10]$, show a decreasing coupling constant for small momenta. Using an asymmetric lattice allow us to study smaller momenta having in mind to provide, at least, a hint to this puzzle.

Again, our lattice data show finite volume effects, if one compares pure temporal and pure spatial momenta, see figure 5 (left). Comparing the results for all available lattices (plane-wave source), see figure 5 (right), we can see, as in the ghost case, finite volume effects, and clear Gribov copies effects.

In what concerns the infrared behaviour, we tried to fit the lowest momenta to a pure power law, $\left(q^{2}\right)^{\kappa_{\alpha}}$. We concluded that this power law is only compatible with the data from $16^{3} \times 128$ lattice, gauge fixed with CEASD method, giving $\kappa_{\alpha} \sim 0.688$, with $\chi^{2} /$ d.o.f. $\sim 0.011$. The reader should be aware that it is also possible, in some cases, to fit the infrared data to $\alpha(0)\left(1+a q^{2}+\ldots\right)$ and get a $\alpha(0) \neq 0$. Therefore, we can not give a definitive answer about the behaviour of the running coupling constant for $q=0$. Note, however, that $\alpha_{S}\left(q^{2}\right)$ for the smallest momenta, seems to increase as a function of the volume.

\section{Future work}

Currently, we are engaged in improving the statistics for our larger lattices and the extrapolations to the infinite volume limit for the gluon and ghost propagators. Furthermore, we plan to perform simulations with larger lattices and, hopefully, combine all our results to provide a reliable answer on the behaviour of the infrared QCD Green's functions. 


\section{Acknowledgements}

The speaker would like to thank Fundação Luso-Americana para o Desenvolvimento (FLAD), Fundação Calouste Gulbenkian and University of Arizona Foundation to make this interesting conference possible to him. This work was supported by FCT via grant SFRH/BD/10740/2002, and project POCI/FP/63436/2005.

\section{References}

[1] C. Lerche, L. von Smekal, Phys. Rev. D65 (2002) 125006 [hep-ph/0202194].

[2] J. M. Pawlowski, D. F. Litim, S. Nedelko, L. von Smekal, Phys. Rev. Lett. 93 (2004) 152002 [hep-th/0312324].

[3] C. S. Fischer, H. Gies, JHEP 0410 (2004) 048 [hep-ph/0408089].

[4] D. Zwanziger, Phys. Rev. D67 (2003) 105001 [hep-th/0206053].

[5] C. S. Fischer, M. R. Pennington, Phys. Rev. D73 (2006) 034029 [hep-ph/0512233].

[6] O. Oliveira, P. J. Silva, AIP Conf. Proc. 756 (2005) 290 [hep-lat/0410048].

[7] P. J. Silva, O. Oliveira, PoS (LAT2005) 286 [hep-lat/0509034].

[8] O. Oliveira, P. J. Silva, PoS (LAT2005) 287 [hep-lat/0509037].

[9] P. J. Silva, O. Oliveira, Phys. Rev. D74 (2006) 034513 [hep-lat/0511043].

[10] O. Oliveira, P. J. Silva, hep-lat/0609027.

[11] O. Oliveira, P. J. Silva, hep-lat/0609036.

[12] A. Cucchieri, T. Mendes, Phys. Rev. D73 (2006) 071502 [hep-lat/0602012].

[13] Andre Sternbeck, PhD thesis, hep-lat/0609016.

[14] R. Alkofer, W. Detmold, C. S. Fischer, P. Maris, Phys. Rev. D70 (2004) 014014 [hep-ph/0309077].

[15] A. Cucchieri, Nucl. Phys. B508 (1997) 353 [hep-lat/9705005].

[16] A. Sternbeck, E.-M. Ilgenfritz, M. Müller-Preussker, A. Schiller, Phys. Rev. D72 (2005) 014507 [hep-lat/0506007].

[17] O. Oliveira, P. J. Silva, Comp. Phys. Comm. 158 (2004) 73 [hep-lat/0309184].

[18] H. Suman, K. Schilling, Phys.Lett. B373 (1996) 314 [hep-lat/9512003].

[19] C. S. Fischer, B. Gruter, R. Alkofer, Annals Phys. 321 (2006) 1918 [hep-ph/0506053].

[20] D. B. Leinweber, J. I. Skullerud, A. G. Williams, and C. Parrinello, Phys. Rev. D60(1999) 094507; Phys. Rev.D61 (2000) 079901 [hep-lat/9811027].

[21] S. Furui, H. Nakajima, Phys. Rev. D70 (2004) 094504 [hep-lat/0403021].

[22] E.-M. Ilgenfritz, M. Müller-Preussker, A. Sternbeck, A. Schiller, I. L. Bogolubsky, hep-lat/0609043.

[23] G. M. Prosperi, M. Raciti, C. Simolo, hep-ph/0607209.

[24] A. Cucchieri, T. Mendes, A. Taurines, Phys. Rev. D71, 051902(R) (2005) [hep-lat/0406020].

[25] A. Sternbeck, E.-M. Ilgenfritz, M. Müller-Preussker, A. Schiller, I. L. Bogolubsky, PoS (LAT2006) 076 [hep-lat/0610053]. 